\title{
Static Output Feedback Control of Flexible Structures subject to Random Disturbances
}

\author{
Motofumi HATTORI, Satoshi TADOKORO, Toshi TAKAMORI \\ Kobe University, Faculty of Engineering \\ (1-1 Rokkodai,Nada,Kobe 657 Japan) \\ Tel. +81-78-803-1200, Fax. +81-78-803-1217 \\ E-mail: hattori@in.kobe-u.ac.jp
}

\begin{abstract}
In this report, we propose a theoretical essence of the stabilization of flexible structures by using the static output feedback control. This shows that not only strain outputs but also other outputs are valid in this control method for flexible structures of many boundary conditions including cantilevers, free ends, and other general boundary conditions. Sufficient conditions of structures and observation mechanisms are obtained for the closed loop systems to be asymptotically stable. When the observation mechanism is nicely chosen, the closed loop system becomes exponentialy stable.
\end{abstract}

Keywords : Distributed parameter sysems, Flexible structures, Flexible robot arms, Flexible manipulators, Direct strain feedback

\section{Introduction}

The direct strain feedback control of flexible structures is the one of the static output controls [6]. The stabilization of distributed parameter systems using static output feedback control are studied by many researchers[2][3] [4][5].

In all these previous papers, observation operators which generate outputs are bounded. But in the above static strain feedback control, output operators are unbounded. In this problem we show that unbounded output operators are transformed to bounded output operators. (This point is not described in the previous papers.) This transformation is valid for not only strain output but also other unbounded outputs. The theoretical essence of the static strain output feedback control is extended to other kinds of outputs. Thus, we can introduce static GENERAL output feedback control which contains the direct strain feedback control.

\section{Dynamics and Observation Mechanisms of Flexible Struc- tures}

Consider a flexible structure and let $u_{1}(t, x) \in R^{q}(q=1,2,3)$ is an elastic displacement at time $t$, position $x \in \Omega$ where $\Omega$ represents a domain of structures and $\Omega \subset R^{p}(p=1,2,3)$. In general, the displacement $u_{1}(t, x)$ of a flexible structure at time $t$ at position $x$ satisfies a partial differential equation

$$
\frac{\partial^{2} u_{1}}{\partial t^{2}}+D_{x} \frac{\partial u_{1}}{\partial t}+A_{x} u_{1}(t, x)=f_{2}(t, x)+w_{(s y s) 2}(t, x)
$$

for suitable partial differential operators $D$ and $A$. For fixed time $t$, the spatial function $u_{1}(t, \cdot)$ can be thought as an element of a suitable Hilbert space $H$. The operator $A$ represents stiffness and $D$ represents damping of flexible structures. $f_{2}(t, x)$ is a control input, and $w_{(s y s) 2}(t, x)$ is a disturbance to the system (the flexible structure). 
By introducing $u_{2}(t, x)=\partial u_{1} / \partial t$, the dynamics Eq.(1) of the flexible structure becomes

$$
\frac{\partial}{\partial t}\left(\begin{array}{l}
u_{1}(t, x) \\
u_{2}(t, x)
\end{array}\right)=\left(\begin{array}{l}
u_{2}(t, x) \\
-A_{x} u_{1}(t, x)-D_{x} u_{2}(t, x)
\end{array}\right)+\left(\begin{array}{l}
0 \\
f_{2}(t, x)
\end{array}\right)+\left(\begin{array}{l}
0 \\
w_{(s y s) 2}(t, x)
\end{array}\right)
$$

Let $u(t, x)=\left(u_{1}(t, x), u_{2}(t, x)\right)^{T}, f(t, x)=\left(0, f_{2}(t, x)\right)^{T}, w_{s y s}(t, x)=\left(0, w_{(s y s) 2}(t, x)^{T}\right.$ and

$$
\begin{array}{r}
\mathcal{A}_{x} u(x)=\left(u_{2}(x),-A_{x} u_{1}(x)-D_{x} u_{2}(x)\right)^{T} \\
\text { for } u(x)=\left(u_{1}(x), u_{2}(x)\right)^{T}
\end{array}
$$

Then, Eq.(2) can be rewritten as

$$
\frac{\partial u}{\partial t}=\mathcal{A}_{x} u(t, x)+f(t, x)+w_{s y s}(t, x)
$$

Consider the following observation mechanism, i.e. suppose we can measure the following data

$$
\begin{aligned}
u_{(o b s)}(t) & =\mathcal{C} u(t, \cdot)+w_{o b s}(t) \\
& =\left(\begin{array}{c}
\left(c_{1}(\cdot), C u_{2}(t, \cdot)\right)_{H} \\
\vdots \\
\left(c_{N}(\cdot), C u_{2}(t, \cdot)\right)_{H}
\end{array}\right)+\left(\begin{array}{c}
w_{(o b s) 1}(t) \\
\vdots \\
w_{(o b s) N}(t)
\end{array}\right)
\end{aligned}
$$

where $w_{o b s}(t)=\left(w_{(o b s) 1}(t) \ldots w_{(o b s) N}(t)\right)^{T}$ is an observation noise, $c_{k}(\cdot)$ is a spatial weighting function $(k=1,2, \cdots, N),(\cdot, \cdot)_{H}$ is an inner product in a Hilbert space $H$, and the observation operator $\mathcal{C}$ is defined as follows.

$$
\mathcal{C} u(\cdot)=\left(\begin{array}{c}
\left(c_{1}(\cdot), C u_{2}(\cdot)\right)_{H} \\
\vdots \\
\left(c_{N}(\cdot), C u_{2}(\cdot)\right)_{H}
\end{array}\right)
$$

\section{CONDITIONS}

We suoopse that

- $C^{-1}$ is a bounded operator on $H$,

- $\tilde{A}=C A C^{-1}$ is a self-adjoint positive definite operator on $H$,

- $\tilde{D}=C D C^{-1}$ is a positive semidefinite operator on $H$, and

- $\tilde{D}=C D C^{-1}$ is positive definite or $(\tilde{\mathcal{C}}, \tilde{\mathcal{A}})$ is observable in infinite dimensional sense.

where

$$
\begin{aligned}
& \tilde{\mathcal{C}}: \mathcal{D}\left(\tilde{A}^{1 / 2}\right) \times H \rightarrow R^{N} \\
& \tilde{\mathcal{C}} y=\left[\left(c_{k}, y_{2}\right)_{H} ; k \downarrow 1,2, \cdots, N\right]
\end{aligned}
$$

and

$$
\begin{aligned}
& \tilde{\mathcal{A}}_{x} y(x)=\left(y_{2}(x),-\tilde{A}_{x} y_{1}(x)-\tilde{D}_{x} y_{2}(x)\right)^{T} \\
& \text { for } y(x)=\left(y_{1}(x), y_{2}(x)\right)^{T} \in \mathcal{D}\left(\tilde{A}^{1 / 2}\right) \times H
\end{aligned}
$$

Examles of $A$ and $C$ which satisfies this condition are as follows. 
Cantilevers When the flexible structure is a cantilever, the spatial domain $\Omega$ of the structure is one-dimensional and $\Omega$ becomes an open interval $(0, L)$ where $L$ is a length of the cantilever. Let $H=L^{2}((0, L) ; R), A_{x}=E I d^{4} / d x^{4}, D=\delta A$, and $C_{x}=d^{2} / d x^{2}$, where $\delta$ and $E I$ are positive constants and

$$
\begin{aligned}
& \mathcal{D}(A)=\left\{u \in H^{4}(0, L) ; 0=u(0)=u^{\prime}(0)=u^{\prime \prime}(L)=u^{\prime \prime \prime}(L)\right\} \\
& \mathcal{D}(C)=\left\{u \in H^{2}(0, L) ; 0=u(0)=u^{\prime}(0)\right\}
\end{aligned}
$$

which reflect the boundary conditions of the both ends $(x=0, L)$ of the structure. $H^{m}(0, L)$ is the Sobolev space of order $m$ on an open interval $(0, L)$ and $E I$ is a positive constant.

Let $c_{k}(x)$ be a positive continuous function which approximates $\delta\left(x-a_{k}\right)(k=$ $1,2, \cdots, N)$. The time derivative of the strains (the bending moments) of the structure at $x=a_{1}, a_{2}, \cdots, a_{N}$ are measured. $A$ and $C A C^{-1}$ becomes positive definite self-adjoint operators on $H$ and the above conditions are satisfied. (See Appendix .)

Free Beams When the flexible structure is a free beam (a beam whose both ends are free), the spatial domain $\Omega$ of the structure is one-dimensional and $\Omega$ becomes an open interval $(0, L)$ where $L$ is a length of the beam. Let $H=L^{2}((0, L) ; R), A_{x}=$ $E I d^{4} / d x^{4}, D=\delta A$, and $C_{x}=d^{2} / d x^{2}$, where $\delta$ and $E I$ are positive constants and

$$
\begin{aligned}
& \mathcal{D}(A)=\left\{u \in H^{4}(0, L) ; 0=u^{\prime \prime}(0)=u^{\prime \prime \prime}(0)=u^{\prime \prime}(L)=u^{\prime \prime \prime}(L)\right\} \\
& \mathcal{D}(C)=\left\{u \in H^{2}(0, L) ; 0=u(0)=u^{\prime}(0)\right\}
\end{aligned}
$$

which reflect the boundary conditions of the both ends $(x=0, L)$ of the structure. $H^{m}(0, L)$ is the $m$-th order Sobolev space on an open interval $(0, L)$ and $E I$ is a positive constant.

Let $c_{k}(x)$ be a positive continuous function which approximates $\delta\left(x-a_{k}\right)(k=$ $1,2, \cdots, N)$. The time derivative of the strains (the bending moments) of the structure at $x=a_{1}, a_{2}, \cdots, a_{N}$ are measured. $A$ and $C A C^{-1}$ becomes positive definite self-adjoint operators on $H$ and the above conditions are satisfied. (The proof is similar to Appendix.)

General Structures The above conditions are satisfied when $A$ is a self-adjoint positive definite operator on the Hilbert space $H, C=A^{\alpha}(0 \leq \alpha \leq 1$ is a constant $)$ and $D=\delta A$ where $\delta$ is a positive constant, since $C A C^{-1}=A$. If $C=A^{\alpha}(0 \leq \alpha \leq$ 1 is a constant), the observed data correspond to time derivatives of the elastic displacements of the structure when $\alpha=0$, to the time derivative of the strains when $\alpha=1 / 2$, and to the shear forces when $\alpha=3 / 4$.

\section{Statistic Properties of the System Disturbance and the Ob- servation Noises}

Suppose that the disturbance $w_{\text {sys }}(t, \cdot)$ to the system and the noise $w_{\text {obs }}(t)$ of the observation mechanism are generated by a cylindrical Brownian motion ( $H$-Brownian motion) $B_{\text {sys }}(t)$ (whose time derivative is a spatially and temporally white noise) and a standard Brownian motion $B_{\text {obs }}(t)$ respectively, i.e.,

$$
\begin{aligned}
d w_{s y s}(t) & =H_{\text {sys }} w_{\text {sys }}(t) d t+Q d B_{\text {sys }}(t) \\
d w_{o b s}(t) & =H_{\text {obs }} w_{\text {obs }}(t) d t+R d B_{\text {obs }}(t)
\end{aligned}
$$


where $H_{\text {sys }}$ is a stable bounded linear operator on a Hilbert space $H$. That is, the all real parts of spectra of the operators $H_{s y s}$ are smaller than some negative constant. $Q$ is a Hilbert-Schmidt operator on a Hilbert space $H, H_{o b s}$ is a stable matrix, and $R$ is a matrix.

Then, the extended system becomes as follows.

$d\left(\begin{array}{l}u(t) \\ w_{\text {sys }}(t) \\ w_{\text {obs }}(t)\end{array}\right)=\left(\begin{array}{ccc}\mathcal{A} & I & 0 \\ 0 & H_{\text {sys }} & 0 \\ 0 & 0 & H_{\text {obs }}\end{array}\right)\left(\begin{array}{l}u(t) \\ w_{\text {sys }}(t) \\ w_{\text {obs }}(t)\end{array}\right) d t+\left(\begin{array}{l}f(t) \\ 0 \\ 0\end{array}\right) d t+\left(\begin{array}{l}0 \\ Q d B_{\text {sys }}(t) \\ R d B_{\text {obs }}(t)\end{array}\right)($

\section{Static Output Feedback Control of Flexible Structures}

Consider a static output feedback control

$$
f(t, x)=G(x) u_{o b s}(t)
$$

where the feedback gain $G(x)$ is

$$
G(x)=-\tilde{g}\left(\begin{array}{c}
0, \cdots, 0 \\
\left(C^{-1} c_{1}\right)(x), \cdots,\left(C^{-1} c_{N}\right)(x)
\end{array}\right)
$$

where $\tilde{g}$ is a positive constant.

By this static output feedback, we obtain the following closed loop system.

$$
d\left(\begin{array}{l}
u(t) \\
w_{\text {sys }}(t) \\
w_{\text {obs }}(t)
\end{array}\right)=\left(\begin{array}{ccc}
\mathcal{A}+G \mathcal{C} & I & G \\
0 & H_{\text {sys }} & 0 \\
0 & 0 & H_{\text {obs }}
\end{array}\right)\left(\begin{array}{l}
u(t) \\
w_{\text {sys }}(t) \\
w_{\text {obs }}(t)
\end{array}\right) d t+\left(\begin{array}{l}
0 \\
Q d B_{\text {sys }}(t) \\
R d B_{\text {obs }}(t)
\end{array}\right)
$$

If we can choose $G$ such that $\mathcal{A}-G \mathcal{C}$ is sufficiently stable, then the variance of the extended stste variable $\left(u(t), w_{\text {sys }}(t), w_{o b s}(t)\right)$ becomes small.

\section{Exponential Stability of the Transformed Closed Loop Op- erator and the Variance of the State Variable of the Closed Loop System}

When neither system disturbances nor observation noises exist, i.e. $w_{s y s}(t, x) \equiv 0$ and $w_{o b s}(t) \equiv 0$, the closed loop system becomes

$$
\begin{aligned}
& \frac{\partial}{\partial t}\left(\begin{array}{l}
u_{1}(t, x) \\
u_{2}(t, x)
\end{array}\right)= \\
& \left(\begin{array}{l}
u_{2}(t, x) \\
-A_{x} u_{1}(t, x)-D_{x} u_{2}(t, x)
\end{array}\right)-\left(\begin{array}{l}
0 \\
\dot{g} \sum_{k=1}^{N}\left(C^{-1} c_{k}\right)(x)\left(c_{k}(\cdot), C u_{2}(t, \cdot)\right)_{H}
\end{array}\right)
\end{aligned}
$$

Introduce a new variable $y_{i}(t)=C u_{i}(t)(i=1,2)$ and $y(t)=\left(y_{1}(t), y_{2}(t)\right)^{T}$, the closed loop system Eq.(18) is rewritten as follows.

$$
\begin{aligned}
& \frac{\partial}{\partial t}\left(\begin{array}{l}
y_{1}(t, \cdot) \\
y_{2}(t, \cdot)
\end{array}\right)= \\
& \left(\begin{array}{l}
y_{2}(t, \cdot) \\
-\tilde{A} y_{1}(t, \cdot)-\tilde{D} y_{2}(t, \cdot)
\end{array}\right)-\left(\begin{array}{l}
0 \\
\dot{g} \sum_{k=1}^{N} c_{k}(\cdot)\left(c_{k}(\cdot), y_{2}(t, \cdot)\right)_{H}
\end{array}\right)
\end{aligned}
$$


where $\tilde{A}=C A C^{-1}$ and $\tilde{D}=C D C^{-1}$.

By defining an operator $\tilde{\mathcal{C}}: \mathcal{D}\left(\tilde{A}^{1 / 2}\right) \times H \rightarrow R^{N}$ as

$$
\tilde{\mathcal{C}} y=\left[\left(c_{k}, y_{2}\right)_{H} ; k \downarrow 1,2, \cdots, N\right]
$$

for $y=\left(y_{1}, y_{2}\right)^{T} \in \mathcal{D}\left(\tilde{A}^{1 / 2}\right) \times H$, the transformed closed loop equation Eq.(19) can be rewritten as

$$
\frac{d}{d t} y(t)=\left(\tilde{\mathcal{A}}-\tilde{g} \tilde{\mathcal{C}}^{*} \tilde{\mathcal{C}}\right) y(t)
$$

The operator $\tilde{\mathcal{A}}-\tilde{g} \tilde{\mathcal{C}} * \tilde{\mathcal{C}}$ in the above equation becomes dissipative on the Hilbert space $\mathcal{D}\left(\tilde{A}^{1 / 2}\right) \times H$. In fact, let $\langle\cdot, \cdot\rangle$ be an inner product in the Hilbert space $\mathcal{D}\left(\tilde{A}^{1 / 2}\right) \times H$, id est,

$$
\begin{aligned}
& \langle u, v\rangle=\left(\tilde{A}^{1 / 2} u_{1}, \tilde{A}^{1 / 2} v_{1}\right)_{H}+\left(u_{2}, v_{2}\right)_{H} \\
& \text { for } u=\left(u_{1}, u_{2}\right)^{T}, v=\left(v_{1}, v_{2}\right)^{T} \in \mathcal{D}\left(\tilde{A}^{1 / 2}\right) \times H
\end{aligned}
$$

then

$$
\left\langle\left(\tilde{\mathcal{A}}-\tilde{g} \tilde{\mathcal{C}}^{*} \tilde{\mathcal{C}}\right) y, y\right\rangle=-\left(\tilde{D} y_{2}, y_{2}\right)_{H}-\tilde{g}(\tilde{\mathcal{C}} y, \tilde{\mathcal{C}} y)_{R^{N}} \leq 0
$$

We show that this system Eq. (19) is asymptotically stable in the Hilbert space $\mathcal{D}\left(\tilde{A}^{1 / 2}\right) \times$ $H$. (This equation looks like the equation in [4]) For the solution $y(t)=\left(y_{1}(t), y_{2}(t)\right)^{T}$ of the closed system Eq.(19), we have

$$
\frac{d}{d t}\langle y(t), y(t)\rangle=-2\left(\tilde{D} y_{2}(t), y_{2}(t)\right)_{H}-2 \tilde{g}(\tilde{\mathcal{C}} y(t), \tilde{\mathcal{C}} y(t))_{H} \leq 0
$$

Therefore, for $\frac{d}{d t}\langle y(t), y(t)\rangle=0$, it is necessary that $y_{2}(t)=0$, since $(\tilde{\mathcal{C}}, \tilde{\mathcal{A}})$ is observable or $\tilde{D}$ is a positive definite operator. This leads to the conclusion that $d y_{2} / d t=0$ and $y_{1}(t)=0$ from Eq.(19). Thus, $\langle y(t), y(t)\rangle$ tends to 0 as $t \rightarrow \infty$. Since $u_{i}(t)=C^{-1} y_{i}(t)(i=$ $1,2)$ and $C^{-1}$ is a bounded operator, $u(t)=\left(u_{1}(t), u_{2}(t)\right)^{T}$ tends to 0 as $t \rightarrow \infty$ and the closed loop system Eq.(18) becomes asymptotically stable.

Moreover, we can show the exponential stability of the transformed closed loop system Eq.(19). In fact, for any eigenvalue $-\mu_{i}^{*}$ of the transformed closed loop operator $\tilde{A}-\tilde{g} \tilde{\mathcal{C}}^{*} \tilde{\mathcal{C}}$ $(i=1,2, \cdots)$,

$$
-\mu_{i}^{*} \leq-\alpha(\grave{g})-\dot{g} \mathfrak{S}(\dot{g})
$$

where

$$
\alpha(\tilde{g})=\min \left\{\left(D \psi_{i 2}^{*}, \psi_{i 2}^{*}\right)_{H} ; i=1,2, \cdots\right\}, \beta(\dot{g})=\min \left\{\sum_{k=1}^{N}\left(c_{k}, \psi_{i 2}^{*}\right)_{H}^{2} ; i=1,2, \cdots\right\}
$$

and $\psi_{i}^{*}$ is the $i$-th normalized eigenfunction of the transformed closed loop operator $\tilde{A}-$ $\tilde{g} \tilde{\mathcal{C}}^{*} \tilde{\mathcal{C}}$.

\section{Conclusions}

In this paper, we found the theoretical essence of static output feedback control of flexible structures. This essence shows that not only strain but also other outputs are effective 
in the stabilization of flexible structures by the static output feedback control, and this control method is applicable for many types of flexible structures including cantilevers and free ends beams and other struciures who has general general boundary conditions. The sufficient conditions (see CONDITIONS) of outputs are clarified for the the static output feedback control to be effective. When the observation mechanism is nicely chosen, the closed loop system becomes exponentially stable.

\section{References}

[1] M.Hattori, S.Tadokoro, and T.Takamori, "Static Output Feedback Control of Flexible Structures (A Stabilizing Control of 2 nd Order Evolution Equations)", The Reports on the 17 th. Young Seminar on Evolution Equations, 1995 (to be published)

[2] R.V.Gressang and G.B.Lamont, "Observers for Systems Characterized by Semigroups", IEEE Trans. Automat. Contr. , vol.AC-20, pp.523-528, 1975.

[3] Y.Sakawa and T.Matsushita, "Feedback Stabilization of a Class of Distributed Systems and Construction of a State Estimator" IEEE Trans. Automat. Contr. , vol.AC20, pp.748-753, 1975

[4] Y.Sakawa," Observability and Feedback Stabilization of Distributed Systems of Hyperbolic Type", SICE Trans. vol.12 no.3,pp.251-256,1976(in Japanese)

[5] T.Nambu,"Stabilizing Compensators for Linear Distributed Parameter Systems", Systems, Control, and Information, vol.38, no.12,pp.666-673,1994 (in Japanese)

[6] Z.H.Luo, "Direct Strain Feedback Control of Flexible Robot Arms : New Theoretical and Experimental Results", IEEE Trans. Automat. Contr. vol.38, pp.1610-1622, 1993

\section{Appendix}

Let $A$ and $C$ be operators on the Hilbert space $H=L^{2}(0, L)$ defined as follows.

$$
\begin{aligned}
& A_{x}=E I \frac{d^{4}}{d x^{4}}, \quad \mathcal{D}(A)=\left\{u \in H^{4}(0, L) ; 0=u(0)=u^{\prime}(0)=u^{\prime \prime}(L)=u^{\prime \prime \prime}(L)\right\} \\
& C_{x}=\frac{d^{2}}{d x^{2}}, \mathcal{D}(C)=\left\{u \in H^{2}(0, L) ; 0=u(0)=u^{\prime}(0)\right\}
\end{aligned}
$$

For any $u \in \mathcal{D}\left(C A C^{-1}\right)$, since

$$
\mathcal{D}(C) \ni A C^{-1} u=\frac{d^{2} u}{d x^{2}}
$$

we have

$$
0=u^{\prime \prime}(0)=u^{\prime \prime \prime}(0) \text { and } u \in H^{4}(0, L)
$$

and since $u \in \mathcal{D}\left(A C^{-1}\right)$, we have $C^{-1} u \in \mathcal{D}(A)$, we have

$$
0=u(L)=u^{\prime}(L)
$$

For $u, v \in \mathcal{D}\left(C A C^{-1}\right)$, by integrating by parts, we have

$$
\left(C A C^{-1} u, v\right)=E I\left(\frac{d^{4} u}{d x^{4}}, v\right)=E I\left(\frac{d^{2} u}{d x^{2}}, \frac{d^{2} v}{d x^{2}}\right)=\left(u, C A C^{-1} v\right)
$$

